\title{
Role of Bone Marrow Trephine Biopsies in the Diagnosis of Hematological and Non-Hematological Disorders
}

\author{
Kricha Pande ${ }^{1,4}$, Arvind Kumar Sinha', Anju Pradhan ${ }^{1}$,Suman Rijal', Nisha \\ Keshari Bhatta ${ }^{3}$ \\ ${ }^{1}$ Department of Pathology, BP Koirala Institute of Health Science, Dharan, Nepal \\ ${ }^{2}$ Department of Medicine, BP Koirala Institute of Health Science, Dharan, Nepal \\ ${ }^{3}$ Department of Pediatrics and adolescent medicine, BP Koirala Institute of Health Science, Dharan, Nepal \\ ${ }^{4}$ Department of Pathology, Nepal Medical College, Kathmandu, Nepal
}

\section{ABSTRACT}

Introduction: Bone marrow examination has become increasingly important for the diagnosis and treatment of hematologic and other illnesses. Bone marrow aspirates along with their peripheral smears and bone marrow trephine biopsies are considered to be complementary.

Materials and Methods: This hospital-based, cross-sectional study was carried out in the Department of Pathology for one year (March 2011 to March 2012). Forty-eight adequate bone marrow aspirates along with peripheral blood smears and trephine biopsies were selected and examined. Bone marrow trephine biopsies performed simultaneously with aspirate were correlated with the other two findings to arrive at a conclusive diagnosis.

Results: Bone marrow aspiration and trephine biopsy were found as complementary tests in $51.8 \%$ of cases. Bone marrow trephine biopsy provided a conclusive diagnosis in $25.9 \%$ of cases where bone marrow aspiration was inconclusive. Bone marrow aspiration alone was diagnostic in $22.2 \%$ of cases. In $43.7 \%$ of cases both aspiration and trephine biopsy were unable to provide a specific diagnosis. Bone marrow biopsy was the diagnostic investigation in cases like aplastic anemia, myelofibrosis, and hypoplastic marrow.

Conclusions: This study concludes that bone marrow aspiration smear along with peripheral smear findings and marrow trephine biopsy is required to arrive at a conclusive result.

Keywords: Anemia; Bone marrow aspiration; Trephine biopsy
Correspondence:

Dr. Kricha Pande, MD

Assistant Professor, Department of Pathology

Nepal Medical College, Teaching Hospital, Jorpati, Kathmandu, Nepal

ORCID ID: 0000-0002-9303-831X

Email: krichapande@gmail.com

Submitted: $6^{\text {th }}$ September 2020 Accepted: $5^{\text {th }}$ December 2020

Source of Support: None

Conflict of Interest: None

Citation: Pande K, Sinha AK, Pradhan A, Rijal S, Bhatta NK. Role of Bone Marrow Trephine Biopsies in the Diagnosis of Hematological and NonHematological Disorders. NMJ 2020;3(2):347-51. DOI 10.3126/nmj.v3i2.31042 


\section{INTRODUCTION}

Bone marrow examination has become increasingly important for the diagnosis and treatment of hematologic and other illnesses. Bone marrow could be examined either by aspiration or by a trephine biopsy. ${ }^{1}$ Useful and much-needed diagnostic, prognostic, and scientific information can be obtained from adequately processed and properly stained bone marrow aspirate and core biopsy specimens. ${ }^{2}$

Bone marrow aspiration is performed to obtain specimens used to assess cellular morphology and to conduct specialized tests on the bone marrow, such as flow cytometry for immunophenotypic analysis, cytogenetic studies, or molecular studies. ${ }^{3}$ Bone marrow aspiration gives little information about the total cellularity of the bone marrow because of the fluctuations in cell counts induced by peripheral blood contamination of the bone marrow specimen, however, an overall impression (e.g. cellular, paucicellular) can be given. In some cases, no marrow can be aspirated resulting in a dry tap where core biopsy plays a vital role. ${ }^{4}$ Bone marrow trephine biopsy is an integral component of the diagnosis, staging, and follow-up of many hematological diseases. ${ }^{5}$

Core needle biopsy of the bone marrow is a safe and useful procedure. It is a valuable diagnostic aid for the evaluation of bone marrow cellularity, fibrosis, infection, or infiltrative diseases. ${ }^{6}$ Trephines are superior to aspirates, especially for the assessment of marrow cellularity, the extent and pattern of tumor infiltration, and the cell type.

Biopsy even provides information regarding anatomic distribution and relationships of hematopoietic cells and other elements such as bony trabeculae, blood vessels, and stroma. ${ }^{4}$ It should not be taken as a substitute for an examination of the marrow by aspiration smear but is a complementary procedure that affords several advantages. ${ }^{6}$

A thorough bone marrow morphologic study involves an examination of peripheral blood smears, direct, particle, and buffy coat bone marrow smears, trephine biopsy imprints, particle and trephine biopsy sections, and marrow volumetric data. ${ }^{8}$

However, bone marrow aspirates along with their peripheral smears and bone marrow trephine biopsies are considered to be complementary. ${ }^{9}$ They are also important in the management of these conditions particularly in the follow-up evaluation of patients undergoing chemotherapy, bone marrow transplantation, and other forms of medical treatment. ${ }^{2}$ Frequently, it is a combination of clues gathered from the examination of several different preparations that leads to a correct diagnosis. ${ }^{8}$

The purpose of this study was to evaluate the role of bone marrow trephine biopsies in the diagnosis of various hematological and non-hematological disorders.

\section{METHODS AND MATERIALS}

This was a hospital-based cross-sectional study, conducted in the Department of Pathology at B.P. Koirala Institute of Health Sciences (BPKIHS), Dharan, Nepal for the period of one year (March 2011 to March 2012). All cellular bone marrow aspirations along with their peripheral smears and all adequate bone marrow trephine biopsies performed at BPKIHS in the Department of medicine and pediatrics were enrolled whereas all the bone marrow aspirations that were diluted with peripheral blood and all inadequate bone marrow trephine biopsies were excluded from the study.

The Bone marrow aspiration procedure was performed using the internationalized technique undertaking all the aseptic precautions. The bone marrow biopsy was obtained through the same skin incision site used for the marrow aspiration. Bone marrow iron and reticulin staining was done in every case and were graded as per the standard grading protocol. ${ }^{4}$

The histopathological findings and the diagnosis made on the trephine biopsies were compared with the findings on the peripheral smears and bone marrow aspirate diagnosis. All peripheral smears were stained with Jenner-Giemsa, all bone marrow aspirates were routinely stained with Jenner-Giemsa along with iron (Perls' stain) and all the marrow trephine biopsies were stained with Hematoxylin and Eosin, Iron and Reticulin stains. A standard protocol was followed for all these staining procedures.

Peripheral blood smears were examined under oil immersion. Each cell type (RBC, WBC, and platelets) was evaluated for both quantitative and qualitative abnormalities.

Bone marrow aspirate was smeared on the glass slides and was examined after fixation and staining with Jenner Giemsa and Perl's stain for haemosiderin. A minimum of 500 nucleated cells was counted under oil immersion magnification. Only intact cells were evaluated and all bare nuclei were excluded. ${ }^{10,11}$

Bone marrow trephine biopsy slides were evaluated for adequacy of the biopsy specimen (approximately $1.5 \mathrm{~cm}$ length was taken as adequate) and for assessment of cellularity and the detection of focal lesions. Focal lesions that would be noted at low power include granulomas and focal infiltrates of lymphoma cells or carcinoma cells. Assessment of bony structure, megakaryocyte numbers, location of cells of erythroid and granulocytic lineages and their relative proportions, and also fungal and protozoal infections were looked for. Abnormal cellular infiltrates blasts, metastatic cells were looked for.

The bone marrow aspiration and biopsies were evaluated and grouped based upon the following critieria:

Group I: BMA was adequate but inconclusive and trephine biopsy was diagnostic.

Group II: Both BMA and Bone marrow biopsy were diagnostic.

Group III: BMA was diagnostic but Bone marrow biopsy was inconclusive.

Group IV: Both were inconclusive.

Group V: BMA was dry tap and bone marrow biopsy was diagnostic.

The obtained data was entered in Microsoft Office Excel 2007 and statistical analysis was done using SPSS 17.0 version. Ethical clearance was taken from the ethical committee of BPKIHS. 


\section{RESULTS}

A total of forty-eight patients with various hematological disorders were included in the study. The age of the patients ranged from 8 months to eighty years old with a mean age of 38 years. The male is to female ratio was $4: 5$. Out of 48 cases, only twenty-seven cases were diagnostic and the remaining twentyone cases had inconclusive reports.

The main indication of bone marrow examination was the evaluation of pancytopenia (37\%), investigation of thrombocytopenia $(25.9 \%)$, anemia $(18.50 \%)$, diagnosis of leukemia/Kala-azar $(7.40 \%)$, to rule out hypereosinophilic syndrome and eosinophilic malignancy $(7.40 \%)$ and pyrexia of unknown origin $(3.70 \%)$. Paleness of the body, gum, and nose bleeding were the most frequent presenting symptoms followed by easy fatigability and weakness whereas pallor was the most frequent clinical finding followed by hepatosplenomegaly and petechial rashes.

Table 1 shows the distribution of various hematological disorders. Nine cases (19\%) were of different types of anemias, six cases $(13 \%)$ were of megakaryocytic thrombocytopenia, four cases $(8 \%)$ were hypoplastic marrow, three $(6 \%)$ were acute leukemia, two cases (4\%) were of myeloproliferative disease and hypereosinophilic syndrome each whereas one case $(2 \%)$ was of secondary thrombocytosis.

Table 1: Distribution of various hematological disorders in forty-eight cases.

\begin{tabular}{ll}
\hline Disorders & No. of cases (n; \%) \\
\hline Anemias & $9(19 \%)$ \\
\hline Megakaryocytic thrombocytopenia & $6(13 \%)$ \\
\hline Hypoplastic marrow & $4(8 \%)$ \\
\hline Acute leukemia & $3(6 \%)$ \\
\hline Myeloproliferative disorders & $2(4 \%)$ \\
\hline Hypereosinophilic syndrome & $2(4 \%)$ \\
\hline Secondary thrombocytosis & $1(2 \%)$ \\
\hline Inconclusive/Descriptive & $21(44 \%)$ \\
\hline Total & $48(100 \%)$ \\
\hline
\end{tabular}

Pancytopenia was seen as one of the main indicators for further assessment of bone marrow in most cases including anemias, leukemias, myelofibrosis, and hypoplastic marrow. (Table 2).

Table 2: Cases presenting as pancytopenia in various hematological disorders

\begin{tabular}{ll}
\hline Disorders & No. of cases (n; \%) \\
\hline Megaloblastic anemia & $4 / 4(100 \%)$ \\
\hline Mixed nutritional deficiency anemia & $2 / 3(67 \%)$ \\
\hline Aplastic anemia & $1 / 1(100 \%)$ \\
\hline Hypoplastic marrow & $4 / 4(100 \%)$ \\
\hline Acute leukemia & $1 / 3(33 \%)$ \\
\hline Myelofibrosis & $1 / 1(100 \%)$ \\
\hline Total & $13 / 16(81 \%)$ \\
\hline
\end{tabular}

\section{Special stains}

Special stains were used routinely in our institute. The advantages of special stains were seen in cases like megaloblastic anemia, myelofibrosis even in chronic myeloid leukemia. The iron grade was increased in all cases of megaloblastic anemia in BMA. One case revealed $1 \%$ blast-like cell in peripheral smear and $8 \%$ blast in bone marrow with Perl's iron grading of $5+$ with inconclusive trephine biopsy. A single case of chronic myeloid leukemia was diagnosed, which was evident in the peripheral smear, marrow aspiration, and trephine biopsy. Reticulin grade was also increased. One case of myelofibrosis was seen. A special stain (Reticulin stain) was done, which showed an increase in reticulin fibres, and thus helped in grading the fibrosis. Thus, trephine biopsy was found to be diagnostically superior in the case of myelofibrosis.

\section{Diagnostic utility of bone marrow trephine biopsies.}

Out of 48 total cases, $51.85 \%$ cases were diagnosed by both aspiration and trephine biopsy whereas in $25.9 \%$ of cases bone marrow trephine biopsy was needed to arrive at a conclusive diagnosis besides bone marrow aspiration. Bone marrow aspiration alone was diagnostic in $22.2 \%$ of cases. In $43.7 \%$ of cases both aspiration and trephine biopsy were unable to provide a specific diagnosis. Table 3 shows the categories into which bone marrow biopsies are grouped.

Table 3: Categorization of Bone marrow findings $(n=48)$.

\begin{tabular}{|c|c|c|c|c|}
\hline Group I & Group II & Group III & Group IV & Group V \\
\hline Hypoplastic marrow $=3(50 \%)$ & Megaloblastic anemia=3 $(22 \%)$ & $\begin{array}{l}\text { Megaloblastic anemia/Refractory } \\
\text { anemia }=1(17 \%)\end{array}$ & $\begin{array}{l}21 \text { cases } \\
(100 \%)\end{array}$ & $\begin{array}{l}\text { Myelofibrosis=1 } \\
(100 \%)\end{array}$ \\
\hline $\begin{array}{l}\text { Hypereosinophilic syndrome }=1 \\
(17 \%)\end{array}$ & $\begin{array}{l}\text { Megakaryocytic } \\
\text { thrombocytopenia=3 }(21 \%)\end{array}$ & Hemolytic anemia=1 $(17 \%)$ & & \\
\hline $\begin{array}{l}\text { Mixed nutritional deficiency } \\
\text { syndrome }=1(17 \%)\end{array}$ & Acute leukemia $=3(22 \%)$ & $\begin{array}{l}\text { Megakaryocytic } \\
\text { thrombocytopenia=3 }(50 \%)\end{array}$ & & \\
\hline \multirow[t]{4}{*}{ Aplastic anemia $=1(16 \%)$} & Chronic myeloid leukemia =1 $(7 \%)$ & $\begin{array}{l}\text { Secondary thrombocytosis=1 } \\
(16 \%)\end{array}$ & & \\
\hline & Hypoplastic marrow=1 $(7 \%)$ & & & \\
\hline & Eosinophilia=1 (7\%) & & & \\
\hline & $\begin{array}{l}\text { Mixed nutritional deficiency } \\
\text { anemia }=2(14 \%)\end{array}$ & & & \\
\hline TOTAL: 6 cases $(22.2 \%)$ & 14 cases $(51.8 \%)$ & 6 cases $(22.2 \%)$ & $\begin{array}{l}21 \text { cases } \\
(43.7 \%)\end{array}$ & 1 case $(3.7 \%)$ \\
\hline
\end{tabular}


BMA alone was diagnostic in cases like megakaryocytic thrombocytopenia, megaloblastic anemia, hemolytic anemia, and secondary thrombocytosis. Bone marrow biopsy was the diagnostic investigation in case of hypoplastic marrow, aplastic anemia, myelofibrosis, hypereosinophilic syndrome, and mixed nutritional deficiency anemia.

The sensitivity of bone marrow aspiration smear over bone marrow trephine biopsy was $63.63 \%$, specificity was $80.76 \%$ with a positive predictive value of $73.6 \%$, the negative predictive value of $72.4 \%$, and accuracy of $72.9 \%$ with the kappa value of agreement revealing moderate strength $(0.44)$ of agreement and was significant statistically with a p-value of 0.004 and Mc Nemar chi-square $p$-value of 0.424 . The sensitivity, specificity, positive predictive value, and negative predictive values are demonstrated in table 4 . The correlation of bone marrow aspiration with bone marrow biopsy was statistically significant.

Table 4: Bone marrow aspiration versus Bone marrow trephine biopsy.

\begin{tabular}{|c|c|c|c|c|c|c|}
\hline \multirow{2}{*}{ BMA } & \multicolumn{2}{|c|}{ BM Bx } & \multirow{2}{*}{ Kappa value } & \multirow{2}{*}{ Mc Nemar chi square test } & \multirow{2}{*}{ P value } & \multirow{2}{*}{ Remarks } \\
\hline & Diagnostic & Descriptive & & & & \\
\hline Diagnostic & 14 & 5 & 0.44 (Good) & \multirow{2}{*}{0.424} & \multirow{2}{*}{0.004} & \multirow{2}{*}{ Sig } \\
\hline Descriptive & 8 & 21 & & & & \\
\hline & Sensitivity $=63.63 \%$ & Specificity $=80.76 \%$ & $\mathrm{PPV}=73.68 \%$ & $\mathrm{NPV}=72.41 \%$ & & \\
\hline
\end{tabular}

\section{DISCUSSION}

The purpose of this study was to evaluate the role of bone marrow trephine biopsies in the diagnosis of various hematological and non-hematological disorders.

The present study was carried out in the department of pathology in association with the department of medicine and pediatrics, BPKIHS, Dharan, and forty-eight cases that were adequate to examine were selected for the study.

The mean age of patients at the time of procedure was 38 years (age range 8 months to 80 years) with a male to female ratio of 4:5. Paleness of the body, gum, and nose bleeding were the most frequent presenting symptoms followed by easy fatigability and weakness whereas pallor was the most frequent clinical finding followed by hepatosplenomegaly and petechial rashes.

Studies done by Ozkalemkas et $\mathrm{al}^{12}$ and Rage et $\mathrm{al}^{13}$ revealed that unexplained cytopenias along with other findings are a strong indicator of the necessity of bone marrow examination which holds true in the present study as pancytopenia was seen in all cases of megaloblastic anemia, aplastic anemia, hypoplastic marrow, and myelofibrosis whereas most cases of mixed nutritional deficiency anemia and even one case of acute leukemia also presented with pancytopenia.

The main indication of bone marrow examination was the evaluation of pancytopenia (37\%) and the most common histopathological diagnosis encountered was anemia (18.70\%). However, this result varied from a study done by Bashawri et $\mathrm{al}^{14}$ which showed that the main indication and diagnosis encountered was acute leukemia.

While aspiration smears provided us with the detailed cellular morphology, biopsy, on the other hand, helped assess marrow cellularity (aplastic anemia, hypoplastic marrow, and mixed nutritional deficiency anemia) and diagnosing cases of myelofibrosis. The bone marrow trephine biopsy was further grouped into five categories for reviewing the diagnostic utility of marrow trephine biopsy which proved that in $51.80 \%$ of cases, bone marrow aspirate and trephine biopsy were complementary. The present findings are comparable to the study done by
Sitalakshmi et al. ${ }^{15}$

The present study revealed that bone marrow aspiration was diagnostic in all the cases of leukemia even when BMA was grossly diluted with blood and trephine provided us with additional information as concluded by Sitalakshmi et $\mathrm{al}^{15}$ and De Wolf-Peeters C.

In the present study, the sensitivity of bone marrow aspiration smear over bone marrow trephine biopsy was $63.63 \%$, specificity was $80.76 \%$ with a positive predictive value of $73.6 \%$, the negative predictive value of $72.4 \%$, and accuracy of $72.9 \%$ with the kappa value of agreement revealing moderate strength (0.44) of agreement and was significant statistically with a p-value of 0.004 and Mc Nemar chi-square p-value of 0.424 .

The study revealed that aspiration was sufficient in making a diagnosis in $14(51.80 \%)$ cases as it correlated well with the diagnosis made on trephine sections and in 7 (25.90\%) cases trephine biopsy was necessary to arrive at a conclusive diagnosis whereas Nanda et $\mathrm{al}^{16}$ found out that aspiration alone gave the diagnosis in $88.6 \%$ and trephine biopsy was necessary in $11.4 \%$ of cases.

Both bone marrow aspirate and trephine biopsies were complementary in all three cases of megaloblastic anemia, three cases of megakaryocytic thrombocytopenia, three cases of acute leukemia, two cases of mixed nutritional deficiency anemia, one case each of hypoplastic marrow, eosinophilia, and chronic myeloid leukemia. Gupta et $\mathrm{al}^{17}$, Moid et $\mathrm{al}^{9}$ also concluded that peripheral smears, BMA, and trephine biopsy are complementary tests in hematology.

Bone marrow aspirate smear alone was able to provide a diagnosis of megakaryocytic thrombocytopenia, megaloblastic anemia, mixed nutritional deficiency anemia, and acute and chronic leukemia whereas trephine biopsy was required in cases of myelofibrosis as seen with the study of Nanda et al. ${ }^{16}$

The statistically significant p-value of bone marrow aspiration and bone marrow trephine biopsy indicated that none of the following test done alone is superior to any of them than when performed in combination. 


\section{CONCLUSIONS}

The decision to perform a marrow aspiration alone or in combination with biopsy depends on the diagnosis being considered. Thus, the present study concludes that histopathological evaluation of bone marrow trephines will not replace the morphological and quantitative data obtained from bone marrow smears and peripheral smear but offers the possibility of adding complementary data.

\section{REFERENCES}

1) Riley RS, William D, Ross M, Zhao S, Chesney A, Clark BD. Bone marrow aspirate and biopsy: a pathologist's perspective. II. Interpretation of the bone marrow aspirate and biopsy. J Clin Lab Anal 2009; 23(5): 259-307. Crossref

2) Anwarul I. Bone marrow aspiration before bone marrow core biopsy using the same bone marrow biopsy needle: a good or bad practice? J Clin Pathol 2007; 60: 212-15.

3) Malempati S, Joshi S, Lai S, Braner DAV, Tegtmeyer K. Videos in clinical medicine: Bone Marrow Aspiration and Biopsy. N Engl J Med. 2009;361(15):e28. Crossref

4) Greer JP, Foerster J, Lukens JN, Rodgers GM, Paraskevas F, Glader B. Wintrobe's Clinical Hematology. Eleventh ed. Philadelphia: Lippincott Williams and Wilkins; 2003, Chapter 1. Examination of blood and bone marrow; p. 15-18.

5) Wilkins BS, Clark DM. Making the most of bone marrow trephine biopsy. Histopathology 2009; 55(6): 631-40. Crossref

6) Sabharwal BD, Malhotra V, Aruna S, Grewal R. Comparative evaluation of bone marrow aspirate particle smears, imprints and biopsy sections. J Post grad Med 1990; 36(4): 194-98. Website

7) De Wolf-Peeters C. Bone marrow trephine interpretation: Diagnostic utility and potential pitfalls. Histopathology 1991; 18 (6): 489-93. Crossref

8) Brynes RK, McKenna RW, Sundberg RD. Bone marrow aspiration and trephine biopsy. An approach to a thorough study. Am J Clin Pathol 1978; 70(5): 753-9. Crossref

9) Moid F, Depalma L. Comparison of relative value of bone marrow aspiration and bone marrow trephine biopsy in diagnosis of solid tumor metastasis and Hodgkin's lymphoma: Institutional experience and literature review. Arch Pathol Lab Med 2005; 129(4): 497-501. Crossref

10) Bain BJ. Bone marrow trephine biopsy. J Clin Pathol 2001; 54: 737 42. Crossref

11) Rindy LJ, Chambers AR. Bone Marrow Aspiration And Biopsy. [Updated 2020 Jun 12]. In: StatPearls [Internet]. Treasure Island (FL): StatPearls Publishing; 2020. Available from: Website

12) Ozkalemkas F, Ali R, Ozkocaman V, Ozcelik T, Ozan U, Ozturk H The bone marrow aspirate and biopsy in the diagnosis of unsuspected nonhematologic malignancy: A clinical study of 19 cases. BMC cancer 2005 ; 1 (5): 144 . Crossref

13) Rege JD, Phatak AM, Roshni Issac, Bichile SK. Experience with Bone marrow trephine biopsy. J Assoc Physicians India 1992; 49: 247-248. Website

14) Bashawri LA. Bone marrow examination. Indications and diagnostic value. Saudi Med J 2002; 23(2): 191-6. Website

15) Sitalakshmi S, Srikrishna A, Devi S, Damodar P, Alexander B. The diagnostic utility of bone marrow trephine biopsies. Ind J Pathol Microbiol 2005; 48(2): 173-176. Website

16) Nanda A, Basu S, Marwaha N. Bone marrow trephine biopsy as an adjunct to bone marrow aspiration. J Assoc Phys India 2002; 50: 893-5. Website

17) Gupta N, Kumar R, Khajuria A. Diagnostic assessment of bone marrow aspiration smears, touch imprints and trephine biopsy in hematological disorders. JK science 2010; 12(3): 130-3. Website 